\title{
Mehrsprachigkeitsdidaktik an deutschen Schulen - eine Zwischenbilanz
}

\section{Zusammenfassung}

Der Großteil aller Schülerinnen und Schüler an deutschen Schulen ist heutzutage mehrsprachig - vor allem durch Migrationshintergründe, bilinguale Elternhäuser und schulischen Fremdsprachenunterricht. Die Mehrsprachigkeitsdidaktik versucht, das Potenzial dieser mehrsprachigen Kompetenzen für den Unterricht in sprachlichen Fächern zu nutzen. Dieser Bericht zieht eine Zwischenbilanz des aktuellen Forschungsstands zu didaktischer Umsetzung, Wirkweisen und Implementation in der Praxis von Mehrsprachigkeitsdidaktik.

Schlüsselwörter: Mehrsprachigkeitsdidaktik, Deutschland, Zwischenbilanz

\section{Multilingual Didactics at German Schools - an Interim Report}

\section{Summary}

Most students at German schools are multilingual nowadays - especially due to migrant backgrounds, bilingual parental homes, and foreign language teaching at school. Multilingual didactics attempts to utilize the potential of these multilingual competencies for language teaching. This article draws up an interim assessment of the current state of research on didactic realization, effects, and practical implementation of multilingual didactics.

Keywords: multilingual didactics, Germany, interim assessment

\section{Mehrsprachigkeitsdidaktik in aller Kürze}

Der Großteil aller Schülerinnen und Schüler an deutschen Schulen ist heutzutage mehrsprachig. Diese Mehrsprachigkeit entsteht vor allem durch Migrationshintergründe, bilinguale Elternhäuser und schulischen Fremdsprachenunterricht. Als mehrsprachig können hierbei nicht nur jene Personen angesehen werden, die auf muttersprachlichem Niveau zwei oder mehr Sprachen beherrschen, sondern auch jene, die weitere Sprachen nur rudimentär erworben haben. Dies wird häufig als funktionale oder funktionelle Mehrsprachigkeit bezeichnet; das heißt, es wird ein Repertoire mit 
unterschiedlich ausgeprägten Fähigkeiten in verschiedenen Kompetenzbereichen (z.B. domänenspezifisch oder mündlich vs. schriftlich) und verschiedenen Sprachen angestrebt, um in unterschiedlichen Situationen sprachlich erfolgreich handeln zu können (vgl. u. a. Europarat, 2001).

In den vergangenen 20 bis 30 Jahren wurden verschiedene Konzepte entwickelt, die zum Ziel haben, diese mehrsprachigen Ressourcen der Lernenden im Sprachunterricht vorteilhaft zu nutzen. Diese Konzepte lassen sich unter dem Begriff Mehrsprachigkeitsdidaktik zusammenfassen und zielen auf den Aufbau einer integrativen Sprachkompetenz ab, zu der alle Sprachen und Sprachlernerfahrungen eines Lernenden beitragen und miteinander interagieren (vgl. ebd., S. 17). Mehrsprachigkeitsdidaktik stellt eine Möglichkeit des Umgangs mit mehrsprachigen Kompetenzen der Schülerinnen und Schüler in sprachlichen Fächern dar. Sie bezeichnet den Unterricht eines Sprachfachs, bei dem die Kompetenzen der Lerngruppe in anderen Sprachen und ihre Sprachlernerfahrungen bewusst einbezogen werden (vgl. Hu, 2010, S. 215). Indem an die sprachlichen Vorerfahrungen und Vorkenntnisse der Schülerinnen und Schüler angeknüpft wird, sollen Synergieeffekte und somit eine Ökonomisierung des Sprachenlernens erreicht werden (vgl. Krumm, 2010, S. 208). Mehrsprachigkeitsdidaktik fungiert hierbei als Transversaldidaktik; das heißt, sie ersetzt die Einzelsprachdidaktiken nicht, sondern flankiert sie (vgl. Meißner, 2004, S. 7). Sie kann in allen sprachlichen Fächern eingesetzt werden, unabhängig davon, ob es sich um Unterricht in einer Muttersprache/Herkunftssprache, einer Zweitsprache oder einer Fremdsprache handelt.

Abb. 1: $\quad$ Ausgangslage und Zielsetzung von Mehrsprachigkeitsdidaktik (=MSD) ${ }^{1}$

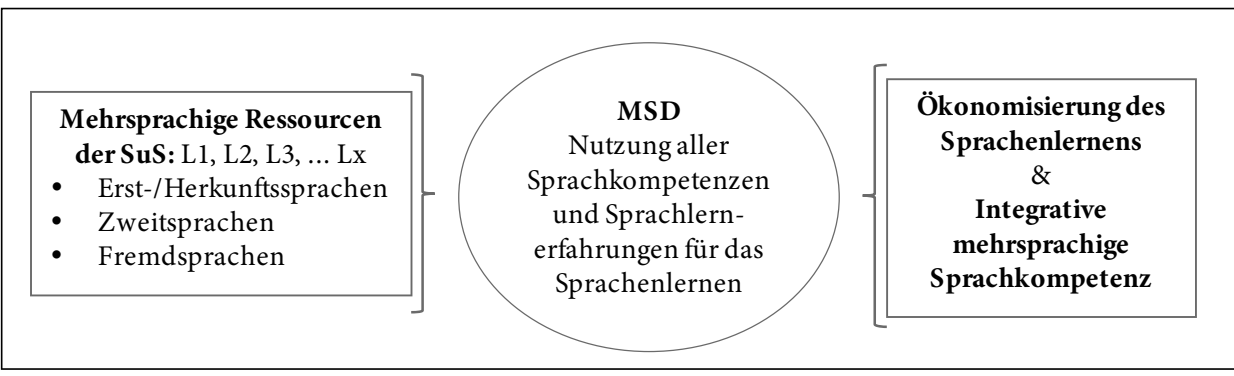

Quelle: eigene Darstellung

\section{Didaktische Umsetzung von Mehrsprachigkeitsdidaktik}

Seit den 1990er-Jahren wurden für die Umsetzung von Mehrsprachigkeitsdidaktik verschiedene Konzepte mit unterschiedlichen Schwerpunktsetzungen entwickelt. Im

1 Einen ausführlichen Überblick über die Bandbreite von Zielsetzungen und Legitimierungsansätzen der Mehrsprachigkeitsdidaktik bieten Reich und Krumm (2013). 
Folgenden werden beispielhaft drei in Deutschland entwickelte Konzepte skizziert, $\mathrm{zu}$ denen auch bereits empirisch gearbeitet wurde, um Gemeinsamkeiten und Unterschiede mehrsprachigkeitsdidaktischer Konzepte aufzuzeigen.

Im Fokus der Interkomprehensionsdidaktik steht der zügige Erwerb der Lesefähigkeit in einer neuen Sprache. Sie wird in erster Linie im Anfangsunterricht einer Fremdsprache eingesetzt, die mit der Umgebungssprache Deutsch und/oder einer oder mehreren bisher erlernten Fremdsprachen der Lernenden verwandt ist (z. B. Englisch und/oder Französisch bei der neu einsetzenden Fremdsprache Spanisch). Der Einstieg in das Erlernen der neuen Sprache erfolgt über die Rezeption authentischer Texte in der Zielsprache. Hierbei sollen Gemeinsamkeiten in Wortschatz und Grammatik als Lernerleichterung dienen. Voraussetzung hierfür ist, dass alle Lernenden über Kompetenzen in mindestens einer der Zielsprache verwandten Sprache verfügen. Der Einbezug unterschiedlicher Herkunftssprachen einer Lerngruppe steht hier nicht im Fokus, sondern meist der Transfer aus bereits gelernten Fremdsprachen und der Umgebungs- und Schulsprache Deutsch. Die Interkomprehensionsdidaktik ist das in Deutschland am weitesten verbreitete mehrsprachigkeitsdidaktische Konzept, zu dem bisher auch die meisten empirischen Studien vorliegen. Als Begründer des Ansatzes gilt Meißner, der ihn zunächst für die romanische Sprachfamilie ausarbeitete (vgl. u.a. Meißner, 1995). Es folgten Erweiterungen für die germanische (vgl. u. a. Hufeisen \& Marx, 2007) und die slawische Sprachfamilie (vgl. u.a. Mehlhorn, 2011). Empirische Studien wurden zunächst im Bereich der Erwachsenenbildung und im Bereich der Hochschullehre durchgeführt; inzwischen liegen auch erste Studien für den schulischen Kontext vor, z.B. die Dissertation von Bär (2009) und die Habilitation von Morkötter (2016).

Beim Konzept des Sprachenübergreifenden Lernens handelt es sich um einen Ansatz, der aus der Interkomprehensionsdidaktik entstanden ist, jedoch einen anderen Schwerpunkt setzt. Hier steht das Ziel im Fokus, die Sprachbewusstheit der Schülerinnen und Schüler durch das Vergleichen von Sprachen zu fördern. Das Konzept wurde für den schulischen Kontext entwickelt und ist nicht auf den Fremdsprachenunterricht beschränkt, sondern kann in allen sprachlichen Fächern sowie auch als fächerübergreifende Projektarbeit eingesetzt werden. Die von der Interkomprehensionsdidaktik abweichende Schwerpunktsetzung bringt es auch mit sich, dass nicht nur Gemeinsamkeiten von Sprachen, sondern auch Unterschiede verstärkt berücksichtigt werden können. Das wiederum führt dazu, dass nicht nur verwandte Sprachen (z.B. Fremdsprachen einer Sprachfamilie), sondern auch heterogene Herkunftssprachen einer Lerngruppe einbezogen werden können (z.B. als Kontrastsprachen oder über die bereits erworbenen Lernstrategien). Entwickelt wurde das Konzept von Behr, die hierzu auch ihre Dissertationsstudie durchführte (2007).

Die Didaktik der Sprachenvielfalt zielt in erster Linie auf den Einbezug der Herkunftssprachen von Schülerinnen und Schülern in den Deutschunterricht, Fremdsprachen 
stehen nicht im Fokus. Sie geht auf den Language-Awareness-Ansatz aus Großbritannien zurück und möchte durch die Erkundung sprachlicher Vielfalt die Neugierde der Lernenden in Bezug auf Sprache und Sprachen wecken. Durch das Nachdenken über Herkunfts- und Zweitsprache im Rahmen von entdeckendem Lernen soll Sprache zum Gegenstand der Aufmerksamkeit werden. Entwickelt und empirisch erprobt wurde dieser Ansatz von Oomen-Welke (vgl. u. a. ebd., 2000).

Auch wenn die Konzepte unterschiedliche Schwerpunkte setzen, so liegen ihnen allen dieselben didaktischen Prinzipien zugrunde. Diese sind vor allem: interlingualen Transfer anzuregen, Sprachbewusstheit zu schulen und Lernstrategien zu vermitteln. Die Gewichtung der Prinzipien kann je nach Ansatz unterschiedlich ausfallen; auch ist eine Verschränkung verschiedener Elemente innerhalb einer Unterrichtssequenz möglich. Darüber hinaus ist in allen Konzepten der Sprachvergleich ein didaktisches Kernelement (vgl. Reich \& Krumm, 2013, S. 103), mit dem diese drei Prinzipien gleichzeitig umgesetzt werden können: Sprachvergleiche können als Lernstrategie angesehen werden und dienen dazu, Transferbasen in anderen gelernten Sprachen aufzuzeigen sowie das metasprachliche Bewusstsein zu fördern. Je nach Lernziel können die dabei einbezogenen Sprachen in den verschiedenen Unterrichtseinheiten variieren; grundsätzlich sollten im Unterrichtsverlauf (z. B. über ein Schuljahr hinweg) jedoch alle Sprachen der Lerngruppe berücksichtigt werden. Auch eignen sich alle sprachlichen Ebenen für Sprachvergleiche, z. B. Wörter (Lexik) oder Wortbildungsverfahren (Morphologie), Wortstellung in einem Satz (Syntax), Wortbedeutungen (Semantik) oder Höflichkeitsformen (Pragmatik) und Merkmale von Textsorten. Schließlich sind Sprachvergleiche nicht auf das Entdecken von Ähnlichkeiten beschränkt, sondern schließen auch das Aufzeigen von Unterschieden mit ein, so dass verwandte Sprachen und Kontrastsprachen gleichermaßen berücksichtigt werden können.

Tab. 1: Umsetzung von Mehrsprachigkeitsdidaktik

\begin{tabular}{|c|c|}
\hline \multicolumn{2}{|c|}{ Umsetzung von Mehrsprachigkeitsdidaktik } \\
\hline Unterrichtsfächer & $\begin{array}{l}\text { - Deutschunterricht } \\
\text { - Fremdsprachenunterricht } \\
\text { - Herkunftssprachenunterricht } \\
\text { - Zweitsprachenunterricht }\end{array}$ \\
\hline Einbezogene Sprachen & $\begin{array}{l}\text { - Erst-/Herkunftssprachen } \\
\text { - Deutsch als Schul-/Umgebungssprache (L1 oder L2) } \\
\text { - Fremdsprachen }\end{array}$ \\
\hline Didaktische Prinzipien & $\begin{array}{l}\text { - interlingualen Transfer anregen } \\
\text { - Sprachbewusstheit schulen } \\
\text { - Lernstrategien vermitteln }\end{array}$ \\
\hline Didaktische Elemente & $\begin{array}{l}\text { - Sprachen vergleichen } \\
\text { - Sprachlernerfahrungen reflektieren } \\
\text { - ... }\end{array}$ \\
\hline
\end{tabular}

Quelle: eigene Darstellung 
Eine der am meisten diskutierten Fragen zur Umsetzung von Mehrsprachigkeitsdidaktik - vor allem in Hinblick auf die Vielfalt der Herkunftssprachen in deutschen Klassenzimmern - besteht darin, wie Lehrkräfte Kompetenzen und Erfahrungen der Lernenden in ihnen selbst unbekannten Sprachen in ihren Unterricht einbeziehen können. Hierzu finden sich in erster Linie die folgenden Vorschläge in der Fachliteratur (für Beispiele vgl. u.a. Brandt \& Gogolin, 2016):

a) die Sprachenvielfalt der Lerngruppe und Sprachlernerfahrungen (z.B. Lerntechniken) der Lernenden zu erfassen und zu berücksichtigen, denn hierfür sind seitens der Lehrkraft keine Kenntnisse in den verschiedenen Sprachen erforderlich;

b) bei Gruppen- und Partnerarbeit den Lernenden freie Wahl für die Arbeitssprache zu lassen, die Ergebnisse jedoch in einer gemeinsamen Sprache der gesamten Lerngruppe präsentieren zu lassen;

c) die Expertise der Schülerinnen und Schüler zu ihren Sprachen (z.B. bei Sprachvergleichen) zu nutzen; im Primarbereich wird oftmals im Rahmen von Hausaufgaben zusätzlich noch die Expertise der Eltern/Familie eingeholt;

d) Kooperationen mit verschiedenen Sprachenfächern einzugehen, z. B. zwischen dem Deutschunterricht und dem herkunftssprachlichen Unterricht; so kann fächer- und sprachenübergreifend dasselbe Unterrichtsthema bearbeitet werden;

e) speziell für Lehrkräfte aufbereitete Informationen $\mathrm{zu}$ den unterschiedlichen Herkunftssprachen zu nutzen, die häufig auch Hinweise auf Unterschiede und Gemeinsamkeiten mit dem Deutschen und mit typischen Fremdsprachen enthalten (vgl. u. a. Krifka et al., 2014; ProDaZ, fortlaufend).

\section{Wirkweisen von Mehrsprachigkeitsdidaktik}

Der Bandbreite an Konzepten und der Vielfalt von dazugehörigen Materialsammlungen steht eine bisher überschaubare empirische Studienlage zu Implementation und Wirkweisen von Mehrsprachigkeitsdidaktik gegenüber. Die durchgeführten Studien fanden anfangs vorwiegend im universitären Umfeld statt; in den letzten zehn Jahren sind nun auch vermehrt Studien im Schulkontext hinzugekommen. ${ }^{2}$ Nachfolgend werden die im Rahmen dieser Studien berichteten Wirkweisen mehrsprachigkeitsdidaktischer Konzepte sowie die Einflussfaktoren auf diese Effekte zusammengefasst. Aufgrund der geringen Studienmenge sind diese Erkenntnisse bisher als Tendenzen zu verstehen.

Mehrsprachigkeitsdidaktischen Ansätzen liegt die Erkenntnis zugrunde, dass mehrsprachige Individuen erhöhte (meta-)linguistische und (meta-)kognitive Fähigkeiten

2 Zu den Studien mit Studierenden zählen u.a.: Meißner (1997), Marx (2005), Meißner (2010), Marx (2010), Zeevaert \& Möller (2011), Bredthauer (2016); Studien mit Schülerinnen und Schülern sind u. a.: Behr (2007; Klasse 7-8), Bär (2009; Klasse 8-10), Bär (2010; Klasse 8), Morkötter (2016; Klasse 4-7). 
entwickeln können, wenn eine entsprechende Förderung stattfindet. Bisherige empirische Studien berichten von Steigerungen in folgenden Bereichen:

1) Die Sprachbewusstheit der Lernenden wird gefördert (vgl. u.a. Marx, 2005, Bär, 2009, Behr, 2007, Meißner, 2010, Bredthauer, 2016). Darunter wird explizites Wissen über Sprache und bewusste Wahrnehmung und Sensibilität beim Sprachlernen, -lehren und -gebrauch verstanden.

2) Die interlinguale Transferfähigkeit der Lernenden, sprich: ihre Kompetenz zum Entdecken von Transferbasen zwischen verschiedenen Sprachen, wird erhöht (vgl. u. a. Marx, 2005, Behr, 2007, Zeevaert \& Möller, 2011, Meißner, 2010, Meißner, 1997).

3) Die Lernleistungen werden gesteigert, in diesem Fall die Sprachkompetenzen der Lernenden in den einzelnen Sprachen - und zwar sowohl in der Zielsprache als auch in den einbezogenen Sprachen. Somit wird von einer Lernerleichterung ausgegangen (vgl. u. a. Marx, 2005, Bär, 2009, Marx, 2010, Bredthauer, 2016).

4) Neben der Sprachbewusstheit und den Sprachkompetenzen werden auch Sprachlernbewusstheit und Sprachlernkompetenz der Lernenden (z.B. Strategieeinsatz) gefördert (vgl. u. a. Bär, 2009, Morkötter, 2016, Behr, 2007, Meißner, 2010, Bredthauer, 2016), sprich: Fähigkeiten in Bezug auf die Lernprozesse. Dies unterstützt die Entwicklung von Lernerautonomie.

5) Verbunden mit den zuvor genannten Effekten wird die Sprachlernmotivation der Lernenden gesteigert (vgl. u. a. Bär, 2009, Behr, 2007, Meißner, 2010, Bär, 2010, Bredthauer, 2016).

6) Durch die erhöhte Lernmotivation steigt auch die Beteiligung und Freude der Lernenden am Unterricht (vgl. u. a. Bär, 2009, Behr, 2007).

7) Nicht nur den Lernenden werden Synergie-Potenziale deutlich, sondern auch das Bewusstsein der Lehrkräfte für Synergieeffekte zwischen den Sprachen wird gefördert (vgl. u. a. Behr, 2007).

Diesen Ergebnissen zufolge können auf mindestens drei Ebenen Wirkweisen von Mehrsprachigkeitsdidaktik angenommen werden: a) Effekte auf die Lernenden (Sprachbewusstheit, interlinguale Transferfähigkeit, Sprachkompetenzen, Sprachlernkompetenz, Sprachlernmotivation), b) Effekte auf die Lehrenden (Bewusstsein der Lehrkräfte für Synergieeffekte) und c) Effekte auf das Unterrichtsgeschehen (Beteiligung und Freude der Lernenden am Unterricht).

Beeinflusst werden die Wirkweisen von Mehrsprachigkeitsdidaktik den Studien zufolge durch folgende Faktoren:

1) Lernerbezogene Faktoren:

- der Kompetenzgrad der Lernenden in der Zielsprache und in den einbezogenen Sprachen und damit verbunden das Maß der mentalen Verfügbarkeit der jeweiligen Sprachen (vgl. u. a. Bär, 2009, Behr, 2007, Meißner, 1997); 
- das Alter der Lernenden und die damit verbundenen generellen kognitiven Fähigkeiten (vgl. u.a. Bär, 2009);

- die Anzahl der von den Lernenden erworbenen Sprachen und die Länge des Erlernens der Sprachen (vgl. u. a. Bär, 2009, Meißner, 1997);

- die Geübtheit der Lernenden im interlingualen Transferieren (vgl. u.a. Bär, 2009, Meißner, 1997) und vermutlich auch in den anderen mit Mehrsprachigkeitsdidaktik verbundenen Lerneraktivitäten;

- die intrinsische Lernmotivation der Lernenden (vgl. u. a. Bär, 2009).

2) Linguistische Faktoren:

- der Grad der typologischen Ähnlichkeit zwischen den einbezogenen Sprachen (in Texten z. B. auch durch die Anzahl an Kognaten/verwandten Wörtern operationalisiert) bzw. die von den Lernenden subjektiv wahrgenommene Nähe der Sprachen (vgl. u.a. Bär, 2009, Marx, 2005, Behr, 2007, Meißner, 2010, Bredthauer, 2016);

- die sprachliche Ebene, da die verschiedenen sprachlichen Ebenen unterschiedlich bewusst sind (vgl. u. a. Bär, 2009, Meißner, 2010, Bredthauer, 2016).

3) Didaktische Faktoren:

- die Fähigkeit der Lehrkraft, die Lernenden für Mehrsprachigkeit zu motivieren (vgl. u. a. Bär, 2009);

- die Moderations-/Beratungskompetenz der Lehrkraft (vgl. u. a. Bär, 2009);

- die Übungsintensität (z.B. des Reflektierens, Vergleichens oder Transferierens) im Unterricht (vgl. u. a. Behr, 2007);

- das Auslösen von Interesse bei den Lernenden durch den Einsatz der Methode des entdeckenden Lernens (vgl. u. a. Behr, 2007).

Abb. 2: Wirkebenen von Mehrsprachigkeitsdidaktik (=MSD) und Einflussfaktoren

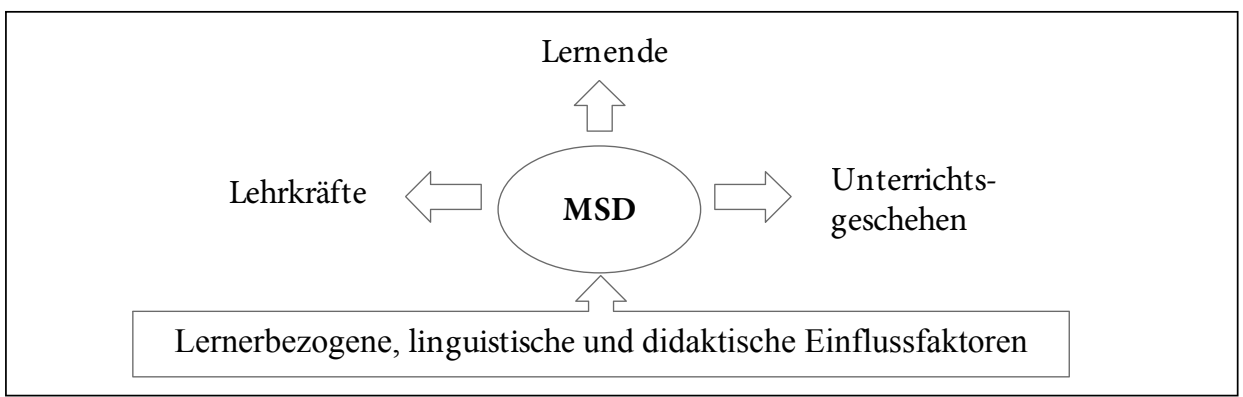

Quelle: eigene Darstellung 


\section{Implementation in der Praxis}

Nicht nur zu den Wirkweisen und Einflussfaktoren von Mehrsprachigkeitsdidaktik, sondern auch zu ihrer bisherigen Implementation in der Bildungspraxis liegen einige Studien vor. Diese kommen zu folgenden Ergebnissen:

\section{Einstellungen der Lehrkräfte und Unterrichtspraxis}

Die Haltungen der Lehrenden gegenüber Mehrsprachigkeitsdidaktik sind in der Regel positiv, aber die Mehrzahl von ihnen hat keine Vorstellung davon, wie eine Umsetzung im Unterricht aussehen kann, oder ist skeptisch hinsichtlich der Realisierbarkeit. Das führt dazu, dass eine Umsetzung von Mehrsprachigkeitsdidaktik im Unterricht der sprachlichen Fächer bisher nur selten stattfindet (zum Überblick vgl. Reich \& Krumm, 2013; Bredthauer \& Engfer, 2016). Um hier eine Veränderung herbeizuführen, bedarf es entsprechender Professionalisierungsmaßnahmen für die Lehrkräfte.

\section{Integration in die Aus- und Fortbildung von Lehrkräften}

Befragungsstudien von Lehrinnen und Lehrern ergeben, dass sie sich meist kaum bis gar nicht darauf vorbereitet fühlen, mehrsprachigkeitsdidaktisch im Unterricht der sprachlichen Fächer zu arbeiten (zum Überblick vgl. Reich \& Krumm, 2013; Bredthauer \& Engfer, 2016). Eine Sensibilisierung für sprachliche Heterogenität und Mehrsprachigkeit im Unterricht ist zwar inzwischen oftmals Bestandteil der Lehramtsstudiengänge, beispielsweise in Form des sogenannten DaZ-Moduls (vgl. u.a. Witte, 2017). Diese bezieht sich jedoch in aller Regel ausschließlich auf sprachsensiblen Fachunterricht, nicht auch auf mehrsprachigkeitsdidaktischen Sprachunterricht. Erste Ansätze zur Integration von Mehrsprachigkeitsdidaktik in die Lehramtsstudiengänge entstehen aktuell. So wurde beispielsweise eine Pilotstudie im Rahmen des DaZ-Moduls in Köln durchgeführt (vgl. Bredthauer, angenommen; Bredthauer, erscheint 2018).

\section{Berücksichtigung in Lehr-/Lernmaterialien}

Eine wesentliche Rolle spielt auch, ob die Lehrenden beim Einbezug der mehrsprachigen Ressourcen ihrer Lernenden in den Unterricht von den zugelassenen Lehrwerken unterstützt werden. In Sprachlehrwerken werden mehrsprachigkeitsdidaktische Ansätze bisher jedoch selten und nur unsystematisch berücksichtigt (vgl. u. a. Marx, 2014). Auf der Grundlage der verschiedenen MehrsprachigkeitsdidaktikKonzepte wurden allerdings verschiedene Materialsammlungen entwickelt, die als Vorbild für eine Integration in Lehrwerke dienen könnten. Einige Beispiele für solche Materialien sind: KIESEL - Kinder entdecken Sprachen für die Klassen 3 bis 8 (ÖSZ), Der Sprachenfächer - Thematische Arbeitshefte (Oomen-Welke \& Arbeitsgruppe, 2006/07 bzw. 2010/11) für die Klassen 4 bis 10, Sprachenvielfalt als Chance - 101 praktische Vorschläge (Schader, 2004) für den Kindergarten bis Klasse 10, Anregungen 
zum sprachenübergreifenden Lernen in der Sekundarstufe I (Behr, 2006) für die Klassen 5 bis 10, Mehrsprachigkeit im Fremdsprachenunterricht. Vergleichen - kombinieren - profitieren (Schöberle, 2015) ab Klasse 8. Diese Materialien werden insgesamt wenig genutzt, konstatiert beispielsweise Oomen-Welke (vgl. 2016, S. 5). Als Erklärungsansatz hierfür wird in Studien verschiedentlich die Bedeutung von Lehrwerken angeführt. Demzufolge betrachten Lehrkräfte Mehrsprachigkeitsdidaktik als etwas Zusätzliches, das der "Abarbeitung von Lehrbuchlektionen“ untergeordnet ist (vgl. u. a. Behr, 2007, S. 161). Dies ließe sich durch eine Öffnung der Lehrwerke für Mehrsprachigkeitsdidaktik ändern, und so lange dies nicht der Fall ist, können Aufgaben aus Lehrwerken oftmals mit geringem Aufwand um den Einbezug der mehrsprachigen Kompetenzen der Lerngruppe erweitert werden. Dies erfordert allerdings wiederum eine entsprechende Aus- bzw. Fortbildung der Lehrkräfte (vgl. u. a. Bredthauer, erscheint 2018).

\section{Bildungspolitische Verankerung}

Die bildungspolitischen Anforderungen an den Unterricht sprachlicher Fächer sind der Unterrichtspraxis in Bezug auf Mehrsprachigkeitsdidaktik schon etwas voraus. So fordern beispielsweise die Bildungsstandards für das Fach Deutsch für den Mittleren Schulabschluss, „Mehrsprachigkeit (Schülerinnen und Schüler mit anderer Muttersprache und Fremdsprachenlernen) zur Entwicklung der Sprachbewusstheit und zum Sprachvergleich [zu] nutzen" (KMK, 2004, S. 16). Doch verglichen mit der Idee eines Gesamtsprachencurriculums, wie sie beispielsweise Hufeisen (2011) oder Ehlich (2017) formulieren, sind auch dies nur erste, kleine Schritte. Mit dieser Idee ist ein „planerische[r] Rahmen, in dem die VertreterInnen der jeweiligen Sprachen (z. B. die Lehrkräfte) untereinander kommunizieren und die VertreterInnen der Sprachenund Sachfächer miteinander arbeiten können" (Hufeisen, 2011, S. 265), gemeint. Für den österreichischen Raum haben Reich und Krumm (2013) sogar ein detailliertes Curriculum der Mehrsprachigkeit entwickelt, das alle Fächer und Schulstufen umfasst; dies kommt der Idee von einem Gesamtsprachencurriculum schon recht nahe. Mit Blick auf die Diskrepanz zwischen bildungspolitischen Anforderungen und unterrichtspraktischer Umsetzung finden sich an verschiedenen Stellen Hinweise darauf, dass sprachenübergreifendes Arbeiten gleichermaßen als Element von Unterrichtsund Schulentwicklung betrachtet werden sollte (vgl. u. a. Behr, 2007, S. 161). Implementationsmaßnahmen zur Mehrsprachigkeitsdidaktik sollten demnach sowohl die Aus-/Fortbildung von Lehrkräften und die Entwicklung/Adaption/Auswahl von geeigneten Unterrichtsmaterialien als auch die Entwicklung von entsprechenden Konzepten auf Schulebene umfassen. 


\section{Desiderata}

Auf der Grundlage der bisherigen Erkenntnisse zur Mehrsprachigkeitsdidaktik und $\mathrm{zu}$ ihrer Implementation lassen sich verschiedene Desiderata formulieren; folgende vier erscheinen von besonderer Relevanz:

- Es liegen zahlreiche mehrsprachigkeitsdidaktische Materialsammlungen, Einzelvorschläge und programmatische Entwürfe vor; allerdings mangelt es bislang an tiefergehenden didaktischen Systematisierungen (vgl. u. a. Reich \& Krumm, 2013, S. 117).

- Ebenso werden weitere empirische Studien zu Wirkweisen und Gelingensbedingungen von mehrsprachigkeitsdidaktisch orientiertem Sprachunterricht benötigt, um die bisherigen Forschungsergebnisse prüfen und ausdifferenzieren zu können (vgl. u. a. Marx, 2005, S. 20).

- Es fehlt bislang an empirisch fundierten Konzepten zur Integration von Mehrsprachigkeitsdidaktik in die Aus- und Fortbildung von Sprachlehrkräften, um diese auf den Einbezug der mehrsprachigen Ressourcen der Schülerschaft in ihren Unterricht vorzubereiten (vgl. u. a. Reich \& Krumm, 2013, S. 130).

- Auf der Grundlage der bisherigen Erkenntnisse zur Mehrsprachigkeitsdidaktik und $\mathrm{zu}$ allgemeinen sprachdidaktischen Erkenntnissen sollte ein Konzept zur Integration von mehrsprachigkeitsdidaktischen Elementen in Lehrwerken entwickelt werden, an dem sich Lehrwerkautorinnen und -autoren sowie Verlage orientieren können (vgl. u.a. Bredthauer \& Engfer, 2016).

\section{Literatur und Internetquellen}

Bär, M. (2009). Förderung von Mehrsprachigkeit und Lernkompetenz - Fallstudien zu Interkomprehensionsunterricht mit Schülern der Klassen 8 bis 10. Tübingen: Narr.

Bär, M. (2010). Motivation durch Interkomprehensionsunterricht - empirisch geprüft. In P. Doyé \& F.-J. Meißner (Hrsg.), Lernerautonomie durch Interkomprehension: Projekte und Perspektiven (S. 281-290). Tübingen: Narr.

Behr, U. (2006). Anregungen zum sprachenübergreifenden Lernen in der Sekundarstufe I (Material Heft 129). Bad Berka: Thüringer Institut für Lehrerfortbildung, Lehrplanentwicklung und Medien.

Behr, U. (2007). Sprachenübergreifendes Lernen und Lehren in der Sekundarstufe I. Tübingen: Narr.

Brandt, H., \& Gogolin, I. (2016). Sprachförderlicher Fachunterricht. Erfahrungen und Beispiele (Förmig Material, Band 8, DVD). Münster et al.: Waxmann.

Bredthauer, S. (2016). Gestaltung, Einsatz und Lernerwahrnehmung mehrsprachigkeitsdidaktischer Elemente im Fremdsprachenunterricht - eine exemplarische Untersuchung in einem universitären Niederländischmodul. Zeitschrift für Angewandte Linguistik, 65 (1), 129-157. https://doi.org/10.1515/zfal-2016-0020.

Bredthauer, S. (erscheint 2018). „Mir war nicht bewusst, welches Potenzial Mehrsprachigkeit für Sprachlernprozesse hat." Anbahnung mehrsprachigkeitsdidaktischer Kompetenzen in der Lehramtsausbildung. In I. Mordellet-Roggenbuck, M. Raith \& K. Zaki (Hrsg.), 
Mehrsprachigkeitsdidaktik an deutschen Schulen - eine Zwischenbilanz |

Mehrsprachigkeit und ihre Didaktik - Modelle und Konzepte für die Lehrer*innenbildung. Frankfurt a. M.: Peter Lang.

Bredthauer, S. (angenommen). „Aber diese verschiedenen Sprachen, die Teil der Klasse waren, wurden nie beachtet." Grundlagen mehrsprachigkeitsdidaktischer Kompetenzen in der Lehramtsausbildung.

Bredthauer, S., \& Engfer, H. (2016). Multilingualism is great - But is it really my business? - Teachers' approaches to multilingual didactics in Austria and Germany. Sustainable Multilingualism, (9), 104-121. https://doi.org/10.7220/2335-2027.9.5.

Doyé, P., \& Meißner, F.-J. (Hrsg.) (2010). Lernerautonomie durch Interkomprehension: Projekte und Perspektiven. Tübingen: Narr.

Ehlich, K. (2017). Ein Gesamtsprachencurriculum für die deutsche Schule des frühen 21. Jahrhunderts: Erforderliche Ziele, absehbare Risiken. In M. Becker-Mrotzek \& H.-J. Roth (Hrsg), unter Mitarbeit von S. Bredthauer \& C. Lahmann, Sprachliche Bildung Grundlagen und Handlungsfelder (S. 249-272). Münster et al.: Waxmann.

Europarat (2001). Gemeinsamer europäischer Referenzrahmen für Sprachen: lernen, lehren, beurteilen. Berlin \& München: Langenscheidt.

$\mathrm{Hu}$, A. (2010). Mehrsprachigkeitsdidaktik. In C. Surkamp (Hrsg.), Metzler Lexikon Fremdsprachendidaktik. Ansätze - Methoden - Grundbegriffe (S. 215-217). Stuttgart: J. B. Metzler.

Hufeisen, B. (2011). Gesamtsprachencurriculum: Weitere Überlegungen zu einem prototypischen Modell. In R. Baur \& B. Hufeisen (Hrsg.), „Vieles ist sehr ähnlich.“ Individuelle und gesellschaftliche Mehrsprachigkeit als bildungspolitische Aufgabe (S. 265-282). Baltmannsweiler: Schneider Verlag Hohengehren.

Hufeisen, B., \& Marx, N. (2007). EuroComGerm - Die sieben Siebe: Germanische Sprachen lesen lernen. Frankfurt a. M.: Shaker.

James, C., \& Garrett, P. (1992). Language Awareness in the Classroom. London \& New York: Longman. https://doi.org/10.1080/09658416.1992.9959804.

KMK (Sekretariat der Ständigen Konferenz der Kultusminister der Länder in der Bundesrepublik Deutschland) (2004). Bildungsstandards im Fach Deutsch für den Mittleren Schulabschluss. Beschluss vom 04.12.2003. München: Luchterhand.

Krifka, M., Blaszczak, J., Leßmöllmann, A., Meinunger, A., Stiebels, B., Tracy, R., \& Truckenbrodt, H. (Hrsg.). (2014). Das mehrsprachige Klassenzimmer. Über die Muttersprachen unserer Schüler. Wiesbaden: Springer VS. https://doi.org/10.1007/978-3-64234315-5.

Krumm, H.-J. (2010). Mehrsprachigkeitsdidaktik. In H. Barkowski \& H.-J. Krumm (Hrsg.), Fachlexikon Deutsch als Fremd- und Zweitsprache (S. 208). Tübingen: Francke.

Marx, N. (2005). Hörverstehensleistungen im Deutschen als Tertiärsprache. Zum Nutzen eines Sensibilisierungsunterrichts im „DaFnE“. Baltmannsweiler: Schneider Verlag Hohengehren.

Marx, N. (2010). Eag and multilingualism pedagogy. An empirical study on students' learning processes on the internet platform English after German. In P. Doyé \& F.-J. Meißner (Hrsg.), Lernerautonomie durch Interkomprehension: Projekte und Perspektiven (S. 225-236). Tübingen: Narr. https://doi.org/10.1080/14790710902972271.

Marx, N. (2014). Häppchen oder Hauptgericht? Zeichen der Stagnation in der deutschen Mehrsprachigkeitsdidaktik. Zeitschrift für Interkulturellen Fremdsprachenunterricht, 19 (1), 8-24.

Mehlhorn, G. (2011). Slawische Sprachen als Tertiärsprachen - Potenziale für den Sprachvergleich im Fremdsprachenunterricht. In B. Rothstein (Hrsg.), Sprachvergleich in der Schule (S. 111-136). Baltmannsweiler: Schneider Verlag Hohengehren. 
Meißner, F.-J. (1995). Umrisse der Mehrsprachigkeitsdidaktik. In L. Bredella (Hrsg.), Verstehen und Verständigung durch Sprachenlernen. Akten des 15. Kongresses für Fremdsprachendidaktik (S. 173-187). Bochum: Brockmeyer.

Meißner, F.-J. (1997). Philologiestudenten lesen in fremden romanischen Sprachen. Konsequenzen für die Mehrsprachigkeitsdidaktik aus einem empirischen Vergleich. In F.-J. Meißner (Hrsg.), Interaktiver Fremdsprachenunterricht (S. 25-43). Tübingen: Narr.

Meißner, F.-J. (2004). Transfer und Transferieren. Anleitungen zum Interkomprehensionsunterricht. In H. Klein \& D. Rutke (Hrsg.), Neuere Forschungen zur Europäischen Interkomprehension (S. 39-66). Aachen: Shaker.

Meißner, F.-J. (2010). Interkomprehension empirisch geprüft: Kompetenzprofile, Mehrsprachenerlebnis, Lernerautonomisierung. In P. Doyé \& F.-J. Meißner (Hrsg.), Lernerautonomie durch Interkomprehension: Projekte und Perspektiven (S. 193-225). Tübingen: Narr.

Morkötter, S. (2016). Förderung von Sprachlernkompetenz zu Beginn der Sekundarstufe. Untersuchungen $z u$ früher Interkomprehension. Tübingen: Narr.

ÖSZ (Österreichisches Sprachenkompetenzzentrum). KIESEL - Kinder entdecken Sprachen. Zugriff am 24.11.2017. Verfügbar unter: www.oesz.at/kiesel/.

Oomen-Welke, I. (2000). Umgang mit Vielsprachigkeit im Deutschunterricht - Sprachen wahrnehmen und sichtbar machen. Deutsch lernen, 25 (2), 143-163.

Oomen-Welke, I. (2016). Mehrsprachigkeit - Language Awareness - Sprachbewusstheit Eine persönliche Einführung. Zeitschrift für Interkulturellen Fremdsprachenunterricht, 21 (2), 5-12. Zugriff am 24.11.2017. Verfügbar unter: http://tujournals.ulb.tu-darm stadt.de/index.php/zif/index.

Oomen-Welke, I., \& Arbeitsgruppe (2006/07). Der Sprachenfächer (Thematische Arbeitshefte). Freiburg i. Br.: Freiburger Verlag. Zugriff am 11.02.2007. Verfügbar unter: www.sprachenfaecher.de. Neu (2010/11): Berlin: Cornelsen.

ProDaZ (fortlaufend). Kontrastive Beschreibungen von häufigen Herkunftssprachen. Zugriff am 24.11.2017. Verfügbar unter: www.uni-due.de/prodaz/einzelsprachen.php.

Reich, H., \& Krumm, H.-J. (2013). Sprachbildung und Mehrsprachigkeit: Ein Curriculum zur Wahrnehmung und Bewältigung sprachlicher Vielfalt im Unterricht. Münster: Waxmann.

Schader, B. (2004). Sprachenvielfalt als Chance. 101 praktische Vorschläge. Zürich: Orell Füssli.

Schöberle, W. (2015). Mehrsprachigkeit im Fremdsprachenunterricht. Vergleichen - kombinieren - profitieren. Stuttgart et al.: Klett.

Witte, A. (2017). Sprachbildung in der Lehrerausbildung. In M. Becker-Mrotzek \& H.-J. Roth (Hrsg.), unter Mitarbeit von S. Bredthauer \& C. Lahmann, Sprachliche Bildung Grundlagen und Handlungsfelder (S. 351-363). Münster et al.: Waxmann.

Zeevaert, L., \& Möller, R. (2011). Wege, Irrwege, Holzwege bei der Texterschließung - Empirische Untersuchungen zur germanistischen Interkomprehension. In F.-J. Meißner, F. Capucho, C. Degache, A. Martins, D. Spita \& M. Tost (Hrsg.), Intercomprehension: learning, teaching, research (S. 146-163). Tübingen: Narr.

Stefanie Bredthauer, Dr., geb. 1983, wissenschaftliche Mitarbeiterin am MercatorInstitut für Sprachförderung und Deutsch als Zweitsprache an der Universität zu Köln.

Anschrift: Mercator-Institut für Sprachförderung und Deutsch als Zweitsprache, Universität zu Köln, Triforum, Albertus-Magnus-Platz, 50923 Köln

E-Mail: stefanie.bredthauer@mercator.uni-koeln.de 